\title{
An Insight into Space Weather
}

\author{
Ashish Mishra $^{1 *}$, Mukul Kumar ${ }^{1,2 *}$ \\ ${ }^{1}$ Department of Physics, Indian Institute of Science Education and Research Bhopal, India. \\ ${ }^{2}$ State Key Laboratory of Space Weather, National Space Science Center, \\ Chinese Academy of Sciences, Beijing, China.
}

\begin{abstract}
dai: https://doi.org/10.21467/ajgr.2.1.46-57
* Corresponding Author email:

1 ashu.mishra274@gmail.com

2 mk.cas@yahoo.com

Article History

Received: 27 November 2017

Revised: 26 December 2017

Accepted: 27 December 2017

Published: 27 December 2017

Student(s)

- $\quad$ Ashish Mishra

Academic Year: 2015-16, $1^{\text {st }}$ Semester

Course Level: Bachelor Degree

Course Name: BS-MS dual degree program

Course year: $4^{\text {th }}$ Year $/ 8^{\text {th }}$ Semester

$\operatorname{Mentar}(\mathrm{s})$

- Mukul Kumar
\end{abstract}

\begin{abstract}
The present article gives a brief overview of space weather and its drivers. The space weather is of immense importance for the spaceborne and ground-based technological systems. The satellites, the power grids, telecommunication and in severe conditions the human lives are at risk. The article covers the effects of solar transient activities (e.g. Solar flares, Coronal mass ejections and Solar winds etc.) and their consequences on the Earth's atmosphere. The space weather is the change in the conditions of interplanetary space because of the solar transient activities. We also discussed the importance of the solar wind which is a continuous flow of the charged energy particles from the Sun to the Earth in respect of the space weather. This article also put light on the Sun-Earth connection and effects of the space weather on it. The Earth's magnetosphere, formed by the interaction of solar wind and Earth's magnetic field behaves like a shield for the Earth against the solar wind.
\end{abstract}

Keywords: Space weather, Coronal mass ejection, Solar flare, Solar wind

\section{Space Weather and Sun-Earth Connection}

\subsection{Space Weather}

The term Space weather refers to those time-dependent conditions of space which are harmful to the spaceborne and ground-based technical systems [1]. Hence, those solar activities which change the interplanetary space conditions and are harmful to Earth atmosphere as well are called the space weather [1] - [7]. In the modern era, the human race is being more and more dependent on the technological systems. These technological systems can be located in the space e.g. satellites, navigation, telecommunication etc. or on the ground like power grids. If we talk about the geomagnetic storms, the satellites in space and the power grids on the Earth are supposed to be most sensitive technological systems. According to the US National Space Weather Programme, the definition is: "conditions on the Sun and in the solar wind, magnetosphere, 
ionosphere and thermosphere that can influence the performance and reliability of space-borne and ground-based technological systems and can endanger human life or health" [1].

To understand the space weather, we need to understand the Sun. In general, the solar dynamic processes are accepted as the primary drivers of the space weather. However, the strongest effects on the space weather are being caused by Solar flares and Coronal mass ejections (CMEs). Flares are responsible for producing intense bursts of ultraviolet light and X-rays. They also accelerate the solar energetic particles (SEPs). CME causes the geomagnetic storms by producing the interplanetary structures. The solar wind plays an important role in the space weather. The nature of the solar wind's speed is variable but at the Earth, the speed of solar wind is approximately $400 \mathrm{~km} / \mathrm{s}$. The interaction of solar wind plasma and Earth's geomagnetic field plays a vital role in the formation of the Earth's magnetosphere. The Magnetosphere of the Earth behaves like a shield to the Earth against the solar wind flow around the Earth. The size of the magnetopause found to vary from 5 to 15 Earth radii (RE) upstream of the Earth. The Magnetosphere stretches by around $1000 \mathrm{RE}$ backward at the night side of the Earth, called the Geo tail. When the Sun partially ionizes the uppermost layer of the Earth's atmosphere as well as the mesosphere and thermosphere by the x-ray and ultraviolet radiation, the Ionosphere formed. The space weather research has been on the forefront of space science because of its importance in the technological and human life. There are a few international bodies keeping an eye on the advances and developments of space weather (e.g. Space weather Program of USA; Living with a star, Nasa-USA; Space weather program of European Space Agency etc.)

\subsection{The Sun-Earth Environment and Space Weather}

The Sun-Earth environment refers to space from the surface of Sun to the upper atmosphere of the Earth, including Earth's magnetic field. This is a tough environment which is full of the Sun's electromagnetic radiations and charged particles. The dipolar magnetic field of the Earth protects us from the violent activities of the Sun. It saves us from the charged particles generated and thrown by the Sun towards Earth during the violent solar activities. The Earth's magnetic field makes a cavity around the Earth, this cavity is called the magnetosphere, and this helps to divert the charged particles [8]. The solar wind is a continuous flow of charged particles which starts from the Sun and reaches to the Earth through the interplanetary space. The variations in the density and the speed of solar wind give rise to space weather. This flow pushes the Earth's magnetic field from the Sun-facing side and drags it towards night side [8].

\section{Drivers of Space Weather}

There can be four subdivisions of the dynamical processes which are related to the space weather namely Solar flares, CMEs, Solar wind, and solar energetic particles (SEPs) [3], [4], [9], [10], [11]. The outer atmosphere of the Sun is designed by strong magnetic fields. It is now well accepted that solar transient phenomena such as solar flares and CMEs occurring on the Sun are responsible for disturbances in the interplanetary medium [10], [11]. CMEs interactions with Earth's magnetosphere may cause severe geomagnetic storms [11], while CME-driven shocks can accelerate energetic particles [12] that may have an impact on human technology in space. Solar flares can suddenly change the conductivity of the ionosphere. These flares and CMEs are the building blocks in the formation of plasma conditions in planetary atmospheres and in the interplanetary space of the solar system. Ultra-relativistic particles and interplanetary shocks excited after the huge energy release processes in the solar corona determine the weather in the whole solar system. Following is the list of a few dynamical processes on the Sun.

\subsection{Coronal Mass Ejections (CMEs)}

The coronal mass ejections are accepted as one of the strongest solar activities which affect the space weather near Earth. CMEs is the rapid and massive ejection of mass and magnetic flux from the outer atmosphere of the Sun (Corona) into the interplanetary space. CMEs are closely related to other solar phenomenon's like eruptive prominences and flares [13] - [18]. 
Mishra et al., Adv.J. Grad. Res.; Vol. 2 Issue 1, pp: 46-57, July 2017

It is accepted that prominence and filament eruptions are found to be closely associated with CMEs. There are evidences that more than $80 \%$ of eruptions lead to a CMEs [13]. Their sheared arcade-like structure contains a large amount of plasma (matter) 'frozen-in' magnetic field and their instability can onset a CME very easily. Prominences are found in almost all regions of the Sun i.e., in active regions, in quiet regions and in between active and quiet regions. The frequency of CME eruption is found to be more in the active regions. However, these three regions are closely related to the onset of CME. CMEs release a huge quantity of matter into the interplanetary space mainly consisting of plasma. Measurement from spacecraft and coronagraphs shows that up to $10^{23}$ Maxwell of magnetic flux and $10^{16} \mathrm{~g}$ of mass can be injected into solar wind due to CME. Table 1 shows the approximate energy requirement for the onset of CME. The total energy of the event may exceed $10^{32} \mathrm{erg}[14]$ - [17].

Table 1: Energy requirements for a moderately large CME, opted from Forbes [14]

\begin{tabular}{|l|l|}
\hline Parameter & Value \\
\hline Kinetic energy (CME, prominence, and shock) & $10^{32} \mathrm{erg}$ \\
\hline Heating and radiation & $10^{32} \mathrm{erg}$ \\
\hline Work done against gravity & $10^{31} \mathrm{erg}$ \\
\hline Volume involved & $10^{30} \mathrm{erg}$ \\
\hline Energy density & $100 \mathrm{erg} \mathrm{cm}^{-3}$ \\
\hline
\end{tabular}

This amount of energy is comparable to that of large flares. This energy gives rise to high velocities of CMEs ranging from the order of $10^{1}$ to $10^{3} \mathrm{~km} / \mathrm{s}$. The average velocity of CMEs as observed by the coronagraphs is around $450 \mathrm{~km} / \mathrm{s}$ [14] - [17]. Solar cycles also affect the frequency of CMEs. The frequency of CME eruption varies from 1-2 per week in solar minimum to 1-3 per day during solar maximum [14] [16]. CMEs are supposed to have a characteristic three-part structure [18]: (i) outer bright limb, which is the front leading edge of the CME, (ii) a cavity which is the empty part of CME and this part follows the bright limb and (iii) the bright core which is the central and brightest part of CME. Figure 1 is the image of CME occurred on August 18, 1980 and clearly shows the three-part structure of CME.

There exist a list of models and arguments in association with the origin of CMEs. Initially, it was thought that $\mathrm{CME}$ results from a sudden release of pressure that consisted of magnetic flux, matter and thermal

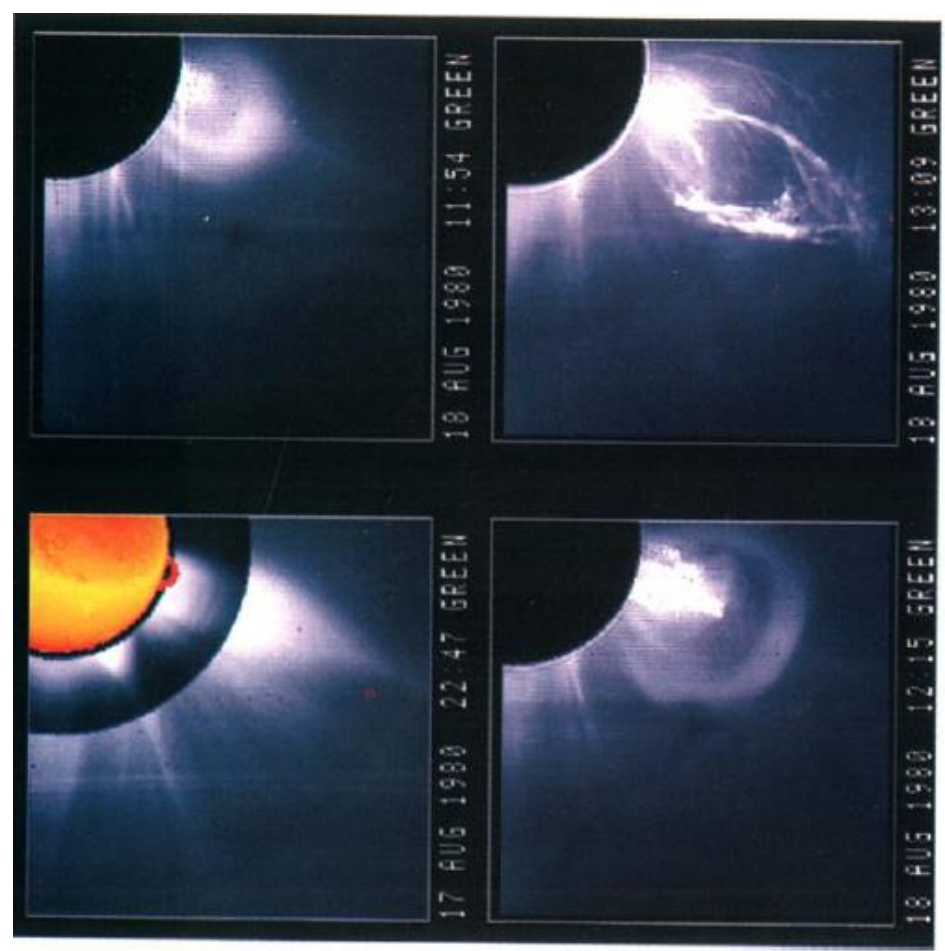

Figure 1: 18 August, 1980 Coronal mass ejection, opted from B.C. Low [18] 
An Insight into Space Weather

energy and it may also be accompanied by eruptive flares and filaments [14], [15]. Van Tend and Kuperus [19] gave a catastrophe mechanism on the onset of CME.

Forbes and Isenberg [20] suggested that the catastrophe mechanism (given by Van Tend and Kuperus, [19]) is applicable in two-dimensional ideal magnetohydrodynamics. They showed that the magnetic reconnection can increase current beyond the limit of equilibrium in the coronal current filament. Due to this increase in current, filament loose equilibrium between magnetic components and tension force and magnetic energy is released. E.R. Priest [21] also suggested that CMEs are triggered by magnetic instability due to magnetic shear or plasma pressure in a magnetic arcade contained by a magnetic medium. This instability gives rise to the eruption of underlying prominence.

Antiochos et al. [22] proposed a 'magnetic breakout' model in which CMEs occur in multi-polar topologies (breakout means reconnection which disrupts the equilibrium of sheared filament channel). In this model, they suggested that CME eruption is triggered by the reconnection between the sheared arcade and neighbouring flux system.

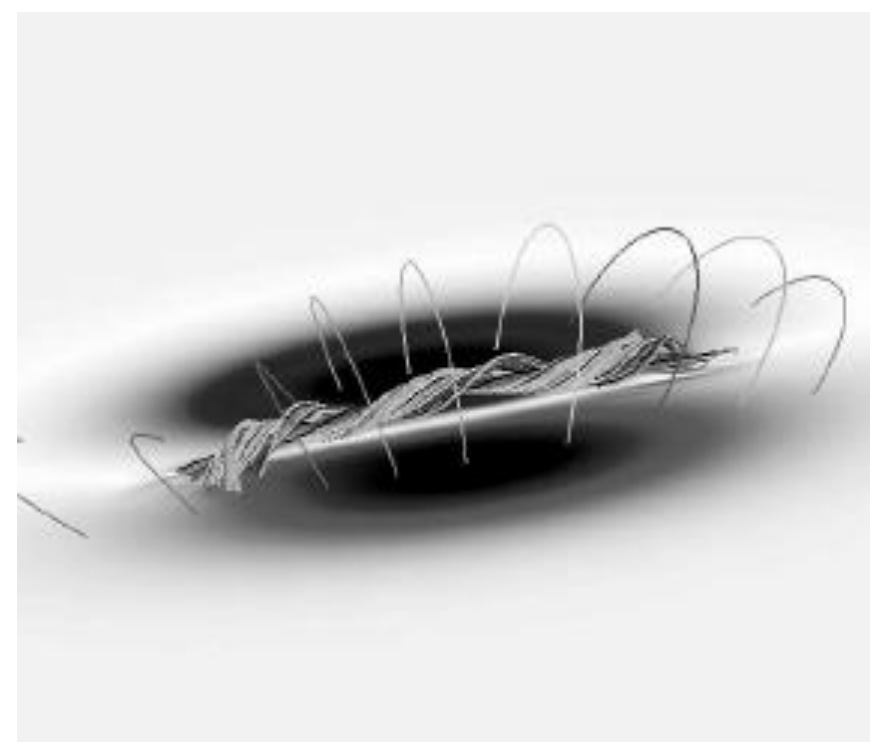

Figure 2: Twisted flux rope, opted from Amari et. al., [24]

Amari et al. [23] gave a theory of large-scale CME onset. In this model, twisted flux rope (Figure 2 shows the topology of the flux rope with helicity) played an important role for magnetic helicity in the corona. They showed that this magnetic structure/configuration loses its stability and a large amount of energy is released during its disruption which onsets a CME. One of the key features of their model was that the energy stored in the pre-eruption phase of the model was so large that it became comparable to the energy of that of open field configuration. Lin and Forbes [25] in their model discussed the importance of the magnetic reconnection process for $\mathrm{CME}$ onset. Even the modest amount of reconnection rates can onset a CME. They suggested that in absence of reconnection, tension force in current sheets become very strong and binds the flux rope so strongly that it does not allow it to erupt and escape into the interplanetary space. They also showed that if a small amount of reconnection can occur then the problem of fully open magnetic field (problem was noted by Aly, [26]) can be avoided.

In respect of CMEs, the importance of gravitational forces has been discussed by B.C. Low [18]. He suggested that the gravitational forces are very important because the average speed of a CME is comparable to the escape velocity of the Sun. The average mass ejection during a CME is of the order of $10^{16} \mathrm{~g}$ and to eject such mass, the work has to be done against the gravity. He also suggested that during a large CME onset, work has to be done against gravity and also a large amount of work is done in kinetic energy of ejected mass. The energy for this work done is provided by the coronal magnetic field. So, in case if it is a small CME, most of the magnetic work is done in lifting the mass against gravity. 
Mishra et al., Adv. J. Grad. Res.; Vol. 2 Issue 1, pp: 46-57, July 2017

Pariat et al. [27] studied 3-dimensional axis-symmetric magnetic reconnection process for generation of solar jets and found that the kink instability can cause a large amount of energy to be released due to magnetic reconnection. They concluded that the energy released is large enough for the generation of jets. Liu et. al. [28] studied and analyzed the observational data of a solar jet event and a CME. They concluded that 3-dimensional magnetic reconnection can trigger solar jet event which can further onset a CME. It is now well understood that the major role of CME is to release magnetic field from stress i.e., to remove magnetic flux and magnetic helicity than to release mass. It is due to instability in magnetic structures that a CME erupts. This eruption releases a large amount of magnetic energy and a significant amount of matter get enough energy to propagate into interplanetary space.

A mechanism for the CME onset and acceleration has been provided by Karpen et al. [29] in their breakout model (breakout means reconnection which disrupts the equilibrium coronal filament channel due to its instability in helicity or sheared structure). Their model suggests that CME eruption triggers due to fast breakout reconnection while the fast acceleration of CME corresponds to fast flare reconnection, not the breakout reconnection. Figure 3 shows the process in three-parts i.e., formation of null point due to reconnection, breakout of current sheet followed by flare current sheet. From their result, they derived a very interesting conclusion that the CME eruption always takes place in filament channel, where enough magnetic energy is stored to erupt and accelerate a CME. This fact shows the clear relationship between the filament channels and CME onsets.
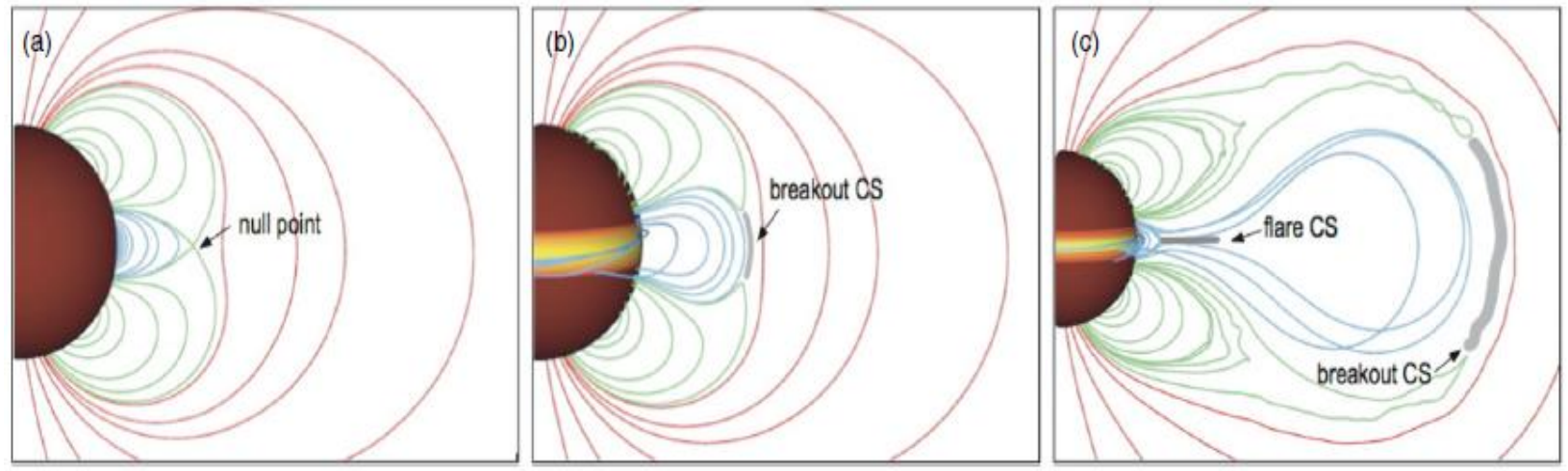

Figure 3: key structures of breakout model using current sheets (CS), opted from Karpen et al., [29]

Most of the prominence eruptions lead to a CME. However, CME can also originate from sunspot regions as those regions have a large amount of magnetic energy stored [16]. In the lower corona, plasma beta i.e., the ratio of thermal pressure and magnetic pressure is very small. It means magnetic energy is the major source of energy in the lower corona and eruptions leads to the release of this energy. When a large amount of matter is released into the planet system during a CME, then it is called interplanetary coronal mass ejection (ICME). ICMEs are always accompanied by solar winds. Interplanetary propagation of CMEs face aerodynamic drag by solar winds and are accelerated or decelerated on the basis of their relative speed with respect to solar wind i.e., the CMEs which are faster than the solar wind are decelerated while the one which has lower speed is accelerated [12], [30] - [33]. The acceleration of CME solely depends on their interaction with solar wind. Since the acceleration of CME largely depends on the amount of energy present at the trigger point, there can be an interaction between two CMEs (which were erupted at different times) and due to this their configuration changes [33].

CME also accelerates the energetic particles emanating from the Sun towards the interplanetary space. These energetic particles interact with the magnetic field of the Earth and along with the solar winds are the major sources of geomagnetic storms [10], [11]. These geomagnetic storms can lead to mass destruction of the assets of Earth e.g. satellite and power grid failures, disruption of communication systems and radiation danger on human beings. 
An Insight into Space Weather

\subsection{Solar Flares}

The study of solar flares goes back to the Carrington White Light Flare, the first one, observed by Carrington in 1859 [34] - [36]. Solar flare phenomenon is one of the dynamical processes occurring in the solar atmosphere. It is often identified by the intense brightness or flash of light which is seen both at the limb and against the disk. The intensity of the flare depends on where it occurs on the Sun. Solar flares emit radiation over the entire electromagnetic spectrum i.e. from long radio waves to very fine gamma rays, see Table 2. Most of the large and violent flares occur in the active region of the Sun which is often followed by CMEs [10], [11], [17].

Table 2: Sources of waves in impulsive flares, [37], [38]

\begin{tabular}{|l|l|}
\hline Waves Produced & Sources \\
\hline Microwaves & $\begin{array}{l}\text { Gyros synchrotron emission from spiraling of } \\
\text { electrons }\end{array}$ \\
\hline Hard X-ray and Gamma ray & $\begin{array}{l}\text { Bremsstrahlung from sub-relativistic and } \\
\text { relativistic electron }\end{array}$ \\
\hline Radio wave (low frequency) & Plasma radiation \\
\hline Soft X-ray & Thermal bremsstrahlung \\
\hline Visible and Extreme Ultraviolet radiation & Hot thermal plasma \\
\hline Gamma ray line & Interaction of MeV ions with ambient nuclei \\
\hline Gamma ray continuum & Decay of pions \\
\hline
\end{tabular}

In spite of having detailed knowledge of the processes of emissions, total energy release during a flare cannot be estimated accurately. It is certainly because of extremely low visibility of flare's fine electromagnetic spectrum against solar disk and energy could not be measured accurately [39], but with the new class of space crafts and solar probes, it now possible to predict energy release within precise limits. It has been proposed by C. L. Wolff [40] that the amount of energy released by a large flare can trigger global solar oscillations. A strong correlation between the appearance of flare and high-frequency solar acoustic spectrum has been established by Karoff et al. [41]. The studies were made by analysing the data obtained from SOHO and GOES satellites. Further, they concluded that solar flare was a driving force for a high-frequency wave. Recently, Kumar et al. [42] analysed the data obtained from VIRGO and GOLF observations and concluded that solar flares can induce global waves on the Sun.

Solar flare phenomenon has always been a fascinating area of research. Initially, according to theory [43], Joule's heating in the chromosphere is not responsible for flare because if it was the case, then only a few metre-thick electric current sheets in a flare would have been set up. Soon with the help of calculation based on magnetohydrodynamics (MHD) and various heating phenomenon going on in Sun's chromosphere, it was made clear that chromosphere heating doesn't contain enough energy which can result in a solar flare. There are evidences [44], [45], which suggest that it is the instability of magnetic field along a neutral line which resulted in a flare. Parker [39] put forward the idea that solar flares are the result of the annihilation of the magnetic field in an active sunspot region. Advances in modern observation techniques led to the development of models of solar flares. Later in 1977, Heyvaerts, Priest and Rust [46] gave a flux emergence model in which they suggested that magnetic flux emergence can give rise to a solar flare. They further suggested that if no greater amount of magnetic energy is stored, it will give rise to a small flare or no flare at all while if a lot of magnetic energy is stored then new flux emerges and may trigger the release of a flare i.e. magnetic environment determines the type of flare. 
Mishra et al., Adv. J. Grad. Res.; Vol. 2 Issue 1, pp: 46-57, July 2017

Yohkoh observations contributed significantly in the development of the magnetic theory of flares. Tsuneta [47] suggested that observed magnetic reconnection at neutral sheet plays a major role in flare energy release. A two dimensional two-ribbon flare model, often known as CSHKP model, named after [48] [51], has been read in detail. This is considered to be a very simplified and almost standard model for flares. In this model, a large loop is considered to be formed with its foot-points fixed. These loops can expand in the solar atmosphere and magnetic reconnection can lead to instability in the loop. This can cause an eruption in the loop and give rise to a flare [48]-[52].

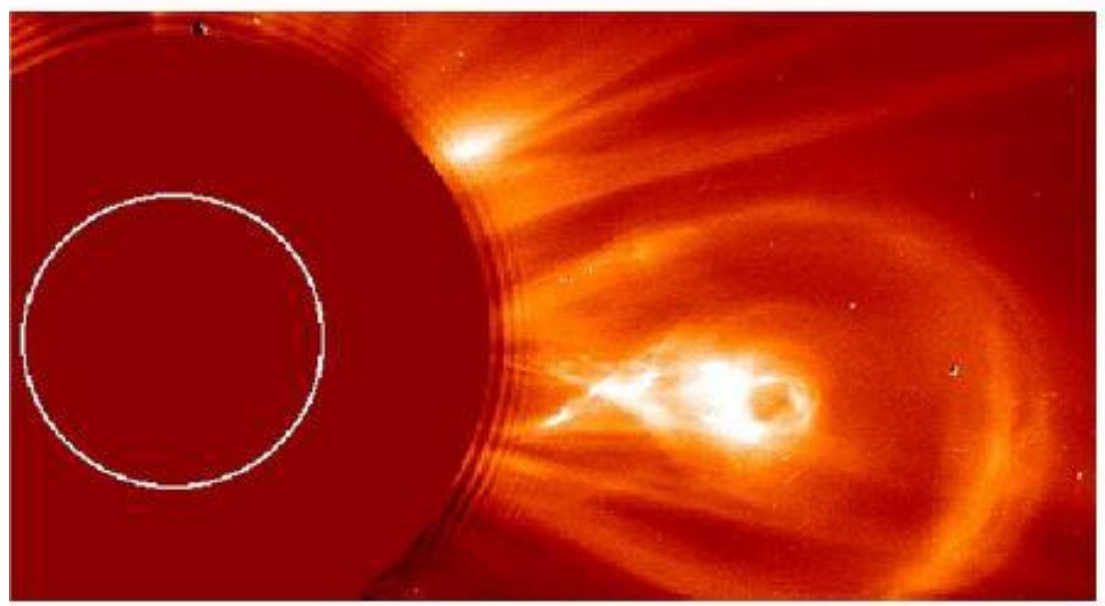

Figure 4: Observation of CME by LASCO/SOHO, opted from Benz [52]

A close relation has been found between the prominences and flares. Figure 4 indicate onset of flare after CME (X-point can be seen behind CME). Martin [53] suggested that flares occur around neutral line within an active region and flares frequently occur in active filament regions. Photospheric motions can release energy into the corona resulting a small flare while a sheared or twisted coronal arcade containing a prominence can lead to magnetic reconnection which can result in a large flare. Priest et al. [38] stressed that storage of free energy and complexity are an important and necessary condition for large solar flares. Considering these conditions, prominence can play a major role in flare eruption as it has a highly sheared magnetic field which implies large storage of free energy. Complexity in prominence allows reconnection and helps to release excess energy which triggers a flare. Sometimes, sunspot motion also increases shear and this shear increases complexity. It may be helpful in triggering the release of energy in an unstable active region structure. It is believed that magnetic field reconnection is responsible for the rapid release of energy which was previously stored as the inductive magnetic fields due to electrical currents flowing into the corona [54]. It is found that free energy in the corona is compatible with that of large flares.

It is now well understood that the flare mostly occurs in the active region where high density strong magnetic field is present near polarity inversion line (PIL) with an excess of magnetic energy. Reconnection model and observations show that CMEs and flares are closely related to each other [55] - [57]. Flares release a large number of solar particles (ions, electrons, atoms etc.) into the interplanetary space. These particles contain very high kinetic energy and it is assumed that these particles are accelerated by shock waves. Some theories also suggest that particle acceleration takes place by Sweet's mechanism. In this mechanism, opposite polarity field lines come close to each other near PIL and magnetic reconnection takes place. Due to this reconnection, energy is released and particles are accelerated into interplanetary space. It is also thought that shock wave mechanism is a developed version of Sweet's mechanism in which shock waves tend to apply force on magnetic field lines of opposite polarity near the neutral line and helps them to reconnect.

It is now well accepted that magnetic field reconnection plays a major role in the solar flare [55]. It is the basic source of flare's energy. This energy is converted to large-scale MHD shock waves, heat and help to accelerate the particles from corona to interplanetary space. This energy accelerates particles to very high 
An Insight into Space Weather

kinetic energy which can reach Earth and can affect it in various ways. These particles can affect satellites, communication systems and can disrupt power grids. They can also lead to some radiation danger for humans.

\subsection{Solar Wind}

The continuous flow of plasma released from the Sun into the interplanetary space and heliosphere is known as solar wind. Solar wind takes away millions of tons of plasma from the Sun every hour and are the major source of mass loss. This plasma is carried away at a very high temperature $\left(\sim 10^{5} \mathrm{~K}\right)$. Solar wind plasma mostly carries protons $\left(\mathrm{H}^{+}\right.$ions) along with alpha particles, however ions of different elements like Carbon, Oxygen, Nitrogen, Iron etc. and electrons are also found. The solar wind is non-uniform and always directed radially away from the Sun and travel with a speed ranging from $250 \mathrm{~km} / \mathrm{s}$ to $800 \mathrm{~km} / \mathrm{s}$ [58], [59].

Solar wind observations show that solar winds can be characterized based on their speed of propagation. Following the characterization, there two types of solar winds: fast solar winds and slow solar winds. Slow solar winds have a velocity ranging from $250 \mathrm{~km} / \mathrm{s}$ to $450 \mathrm{~km} / \mathrm{s}$ with a temperature of the order of $10^{6} \mathrm{~K}$ while the fast solar winds have velocity ranging from $450 \mathrm{~km} / \mathrm{s}$ to $800 \mathrm{~km} / \mathrm{s}$ with the temperature of the order of $10^{5} \mathrm{~K}$ (see Table 3) [58], [59]. Solar high-speed winds catch up the low-speed winds and overall composition of wind changes.

Table 3: Properties of slow and fast solar winds, opted from S.R. Cranmer [59]

\begin{tabular}{|l|c|c|}
\hline Quantity & Slow Wind & Fast Wind \\
\hline Radial Flow Speed & $250-450 \mathrm{~km} / \mathrm{s}$ & $450-800 \mathrm{~km} / \mathrm{s}$ \\
\hline Proton Density (1AU) & $5-20 \mathrm{~cm}^{-3}$ & $2-4 \mathrm{~cm}^{-3}$ \\
\hline Proton Temperature (1AU) & $0.03-0.1 \mathrm{MK}$ & $0.1-0.3 \mathrm{MK}$ \\
\hline Electron Temperature (1AU) & $0.1-0.15 \mathrm{MK}$ & $\sim 0.1 \mathrm{MK}$ \\
\hline Freezing-in Temperature & $1.4-1.7 \mathrm{MK}$ & $1.0-1.3 \mathrm{MK}$ \\
\hline He - abundance & $0.5 \%-4 \%$ & $3 \%-5 \%$ \\
\hline
\end{tabular}

It was observed that irrespective of its direction of motion i.e., either toward the Sun or away from it, comet always had its tail away from the Sun. Biermann [60] pointed out that a comet tail could be always away from the Sun if it encounters with a gas stream flowing away from the Sun.

This conclusion of Biermann was considered and studied by Parker [61] and he named the gas which flows away from the Sun in all directions as "Solar Wind". Further on the basis of his study of magnetohydrodynamics (MHD), Parker [62] showed that the gravitational field is not capable enough to confine the corona due to corona's high thermal energy. He suggested that the solar corona is expanding with appreciable velocity away from the Sun at very large distances. He called this continual outward flow of gas into the interplanetary space as Solar Wind.

Coleman [63] studied the spectral properties of the fluctuations in the solar plasma. He suggested that solar wind was turbulent in regions in 1 Astronomical Unit (1 A.U.). He further suggested that the differential motion of the streams was the major source of energy for the turbulence in the solar wind, e.g., see [64]. Goldstein et al. [65] suggested that the fluctuations in the solar winds occur due to the time variability and inhomogeneous flows. They also suggested that Alfvenic fluctuations are the major component of solar wind turbulence.

Krieger et al. [66] studied the properties of solar wind and the lower corona. They also studied the idea of the origin of high-velocity streams of solar wind from magnetically open regions in the corona. They suggested that the coronal holes are the sources of high-velocity solar wind streams. By their observation of coronal holes in soft X-Ray wavelength, they suggested that in the coronal hole magnetic field is weak and radial while at the boundaries of the hole magnetic field arches away from the hole i.e., the magnetic configuration is divergent at the boundaries of the hole. 
Mishra et al., Adv.J. Grad. Res.; Vol. 2 Issue 1, pp: 46-57, July 2017

They also suggested that this divergent magnetic field is the source of a high-velocity wind stream. Other properties of coronal holes which is consistent with the solar wind are that the coronal density in the holes is significantly lower than that in the closed magnetic field regions and the temperature of this region is lower than that of closed regions. Furthermore, they suggested that the open coronal field configuration would result in the formation of coronal holes.

Nolte et al. [67] from their investigation of the coronal hole and solar wind data concluded that coronal holes are the source of high-velocity solar winds. They examined the coronal hole properties like low density, temperature, reduced X-rays and extreme ultraviolet emissions and weak, open, diverging and unipolar magnetic field region. By analyzing these properties, they found that in coronal hole associated streams, magnetic field polarity below the coronal holes had a good correlation with interplanetary magnetic field polarity.

Tu et al. [68] examined the magnetic structure as the source of fast solar wind in the solar corona. They observed funnel-shaped magnetic configuration which was anchored in the magnetic structures and the solar wind flows coming out from the top of this funnel-shaped magnetic structure with a speed of about $10 \mathrm{~km} / \mathrm{s}$ i.e., the wind flows start in coronal funnels by the magnetic reconnection in the plasma near all sides of these funnels. Studies of low-speed solar winds show that these slow solar winds are related to transient openings of closed coronal magnetic field regions. These unsteady streams lose their equilibrium with the corona and extend outwards into interplanetary space.

McKenzie et al. [69] showed that acceleration of solar wind could not be accounted to pressure gradient from Alfven waves as it acts too slowly that it cannot accelerate rapidly close to the Sun. Cranmer et al. [70] showed that the coronal heating and solar wind acceleration can be associated with MHD Alfven waves. De Pontieu et al. [71] suggested a possible mechanism for solar wind acceleration and coronal heating by Alfven waves. Observations have revealed that the chromosphere contains Alfven waves of very high amplitudes $(10-25 \mathrm{~km} / \mathrm{s})$. The energy calculation of flux carried by these waves has revealed that the Alfven waves have enough energy to accelerate the solar wind and heat the corona.

McIntosh et al. [72] also suggested that high amplitude Alfven waves are the sources of solar wind acceleration and coronal heating. They suggested a two-stage process which is of utmost importance in the quiescent solar atmosphere. In the first stage, injection of plasma from lower corona and heating takes place followed by the second stage in which dissipation of Alfven waves takes place. It was concluded that this dissipation of Alfven waves can account for coronal heating and acceleration of solar winds, and the transfer of energy from low-frequency Alfvenic waves to the plasma is essential to drive the fast solar wind to high velocities at 1 A.U. from the Sun [72]. Wang Y. -M [73] studied the open flux near active region and small coronal holes in active regions and concluded that during solar maximum, these small coronal holes are the source of slow solar wind.

Movement of fast charged particles (cosmic rays) in the solar wind has been studied in detail by many researchers. The continuous presence of solar wind in interplanetary space provides a natural vehicle for many of the cosmic ray effects [74]. Interplanetary magnetic field plays an important role in the motion of cosmic rays in solar winds. The interplanetary magnetic field is irregular and thus scatter the charged particles in interplanetary space very effectively and further, they are carried away by the solar winds. The solar wind flows radially outward in all directions away from the Sun into the interplanetary space.

During its propagation, it interacts with planets, satellites and local interstellar medium. The local interstellar medium contains partially ionized gas [75] and solar winds interact with the local interstellar medium in a very interesting way. As we already discussed, Solar wind contains protons, ion, charged particles while the interstellar medium contains partially ionized gas as well as neutral hydrogen atoms. The neutral hydrogen is coupled weakly to the solar wind plasma via resonant charge exchange - a coupling which leads to the production of pick up ions that come to dominate the internal energy of the solar winds [76]. This increase in energy has important implication for solar wind heating.

Solar winds also interact with planets. Observations show that a shock wave is created ahead of planets on their interaction with solar winds because of its very high velocity. When a planet is not magnetised (Mars, 
An Insight into Space Weather

Venus), the planet itself is an obstacle for the solar winds and the shock is very near to the planet's surface while the other planets, like Earth, which have an intrinsic magnetic field, shock is away from planet's surface [77]. Shockwave is observed ahead of a magnetic field of planets (planet's magnetosphere). The solar wind is the main cause of teardrop shape of Earth's magnetosphere. High-speed solar winds exert high pressure on the magnetosphere and get elongated in the direction opposite to that of Sun, known as the geomagnetic tail.

The non-magnetised solar winds simply flow around the Earth, confining completely the Earth's magnetic field into a cavity [78]. In this closed magnetic field, a large amount of magnetic energy is stored. Magnetosphere deflects the solar wind and does not allow high velocity charged particles to reach Earth. This solar wind compresses magnetosphere very strongly through the interplanetary shock wave. Studies show that this compression can lead to geomagnetic storms as they can suddenly onset the release of stored energy in the magnetosphere. The geomagnetic storm can affect human lives in a variety of ways. They also produce magnificent auroral activities on the poles of Earth, along with disrupting communication systems, satellites, power grid failures etc. In the interplanetary space, solar wind plays a major role as it is the major force acting on all heliospheric and solar system particles, affect interplanetary magnetic field configuration, affect cosmic ray intensity, interacts with local interstellar medium and most importantly, they play a vital role in geomagnetic activities.

\section{Conclusion}

This article presented a brief overview of space weather and its drivers. It has been concluded that the activities in the solar atmosphere like coronal mass ejections, solar flares and solar winds regulate space weather. The filament and prominence eruptions are found to be closely related to coronal mass ejection (CME) and solar flares. Coronal mass ejections throw a huge amount of solar mass, enriching the solar wind with solar high energy particles as well as helps to increase their speed. A solar flare is often recognized by a sudden intense increase in brightness on the solar surface. They are supposed to be one of the most intriguing drivers of space weather. After various models, theories and triggering mechanism of flares, the relation of the flares and CMEs are still on the forefront of solar research. Although the scenario has been changed after the high-resolution spaceborne and ground-based observations still the answers of fundamental problems in flares and CMEs are infancy. Predictions of space weather are of crucial importance to technologically advanced space-age of our civilization. On the other hand, the long-term forecasts of space weather require the knowledge of precursors for solar flares/CMEs. This advancement in our knowledge of solar eruptions and their relation to space weather is mainly due to the current availability of a vast array of space and ground-based observatories.

\section{How to Cite this Article:}

A. Mishra and M. Kumar “An Insight into Space Weather”, Advanced Journal of Graduate Research, vol. 2, no. 1, pp. 46-57, Dec. 2017. doi: 10.21467/ajgr.2.1.46-57

\section{References}

[1] Arnold Hanslmeier, "The Sun and Space Weather", Springer, 2007, ISBN - 9781402056048

[2] Louis J. Lanzerotti, "Space Weather effects on communication", 2002.

[3] D.N. Baker, "Introduction to space weather", Lect. Notes Phys. 656, 3-20, 2005.

[4] M. Moldwin, "Introduction to space weather", 2008, ISBN - 9780521711128

[5] J. -P. Rozelot, Solar and Heliospheric Origins of Space Weather Phenomena, Lect. Notes Phys. 699.

[6] K. Georgieva, "Space weather and Space Climate - What the Look from the Earth Tells Us About the Sun", J.-P Rozelot, C.Neiner (eds), The Environment of The Sun and The Stars, Lecture notes in Physics, 857, 2013.

[7] Hannu E.J. Koskinen, Daniel N. Baker, Andre Balogh, Tamas Gombosi, Astrid Veronig, Rudolph von Steiger, "Achievements and Challenges in the Science of Space Weather", Space Science Rev, 212:1137-1157, 2017.

[8] Kenneth Lang, "The Sun from Space”, Springer, 2000, ISBN - 9783540669449

[9] Spiro K. Antiochos, "Solar Drivers of Space Weather", ASP Conference Series, Volume 95, 1996.

[10] N. Gopalswamy, "History and development of coronal mass ejections as a key player in solar terrestrial relationship", Geoscience Letters, 3:8, 2016. 
Mishra et al., Adv. J. Grad. Res.; Vol. 2 Issue 1, pp: 46-57, July 2017

[11] N. Gopalswamy, "Introduction to special section on large scale geomagnetic storms", Journal of Geophysical Research, Vol. 114, A00A00, 2009.

[12] B. Vrsnak, N. Gopalswamy, "Influence of the aerodynamic drag on the motion of interplanetary ejecta", Journal of Geophysical Research, Vol. 107, No. A2, 1019, 2002.

[13] B. Schmeider, P. Demoulin, G. Aulanier, "Solar filament eruptions and their physical role in triggering coronal mass ejections", Advances in Space Research, Volume 51, Issue 11, 1967-1980, 2013.

[14] T.G. Forbes, "A review on the genesis of coronal mass ejections", Journal of Geophysical Research, Vol. 105, No. A10, 2315323165, 2000.

[15] W.J. Wagner, "Coronal mass ejections", Annual Reviews in Astronomy and Astrophysics, 22, 267-289, 1984.

[16] N. Gopalswamy, S. Akiyama, S. Yashiro, P. Makela, "Coronal mass ejection from sunspots and non-sunspots regions", arXiv: 0903.1087v1 [astro.ph-SR], 2009.

[17] Nat Gopalswamy, "Coronal Mass Ejection and Space Weather", Climate and weather of the Sun and Earth System (CAWSES), Selected papers from the 2007 Kyoto symposium, Edited by T. Tsudu, R. Fujii, K. Shibata, M.A. Geller, pp 77-120, TERRAPUB, TOKYO, 2009

[18] B.C. Low, "Coronal mass ejections, magnetic flux ropes and solar magnetism”, Journal of Geophysical Research, Vol. 106, No. A11, 25141-25163, 2001.

[19] W. VanTend, M. Kuperus, "The development of coronal electric current systems in active regions and their relation to filament and flares", Solar Physics, 59, 115-127, 1978.

[20] T.G. Forbes, P.A. Isenberg, "A catastrophe mechanism for coronal mass ejection”, The Astrophysical Journal, 373, 294-307, 1991.

[21] E.R. Priest, "The initiation of solar coronal mass ejections by magnetic non-equilibrium", The Astrophysical Journal, 328, 848-855, 1988.

[22] S.K. Antiochos, L.R. DeVore, J.A. Klimchuk, "A model for solar coronal mass ejection”, The Astrophysical Journal, 510, 485-493, 1999.

[23] T. Amari, J.F. Luciani, Z. Mikic, J. Linker, "A twisted flux rope model for coronal mass ejections and two ribbon flares", The Astrophysical Journal, 529, 49-52, 2000.

[24] T. Amari, J.F. Luciani, J.J. Aly, Z. Mikic, J. Linker, “Coronal mass ejection: Initiation, magnetic helicity and flux ropes. II. Turbulent diffusion-driven evolution, The Astrophysical Journal, 595, 1231-1250, 2003.

[25] J. Lin, T.G. Forbes, "Effect of reconnection on the coronal mass ejection process", Journal of Geophysical Research, Vol. 105, No. A2, $2375-2392,2000$.

[26] J.J. Aly, "On some properties of force-free magnetic fields in infinite regions of space", The Astrophysical Journal, 283, 349-362, 1984.

[27] E. Pariat, S.K. Antiochos, C.R. DeVore, “A model for solar polar jets”, The Astrophysical Journal, 691, 61-74, 2009.

[28] Jiajia Liu, Yuming Wang, Chenglong Shen, Kai Liu, Zonghao Pan, S. Wong, "A solar coronal jet event triggers a coronal mass ejection", The Astrophysical journal, 813, 115 (6pp), 2015.

[29] J.T. Karpen, S.K. Antiochos, C.R. DeVore, "The mechanism for the onset and explosive eruption of coronal mass ejection and eruptive flares", The Astrophysical Journal, 760, 81(15pp), 2012.

[30] N. Gopalswami, A. Lara, R.P. Lepping, M.L. Kaiser, D. Berdichevsky, O.C. St.Cyr, "Interplanetary acceleration of CMEs", Geophysical Research Letters, Vol. 27, No. 2, 145-148, 2000.

[31] B. Vrsnak, T. Zic, D. Vrbanec, M. Temmer, T. Rollett, C. Mostl, A. Veronica, J. Calogovic, M. Dumbovic, S. Lulic, Y.-J. Moon, A. Shanmugaraju, "propagation of interplanetary coronal mass ejections: The drag based model", Solar physics, 285, 295-315, 2013.

[32] Emilia Kilpua, Hannu E.J. Koshkinen, Tuija I. Pulkkinen, "Coronal mass ejections and their sheath regions in the interplanetary space", Living Reviews in Solar Physics, 14:5, 2017.

[33] Noe Lugaz, Manuela Temmer, Yumig Wang, Charles J. Farrugia, "The interaction of successive coronal mass ejections: A review", arXiv: 1612.02398v2 [physics.space-ph], 2017.

[34] Louis J. Lanzerotti, "Value of Historical Space Weather Events", Space Weather, Vol. 5, S06005, 2007.

[35] Louis J. Lanzerotti, "Space Weather: Historical and Contemporary perspective”, Space Science Rev, 212:1253-1270, 2017.

[36] Ilya G. Usoskin, "A History of Solar Activity over the Millennia", Living Reviews in Solar Physics, 14:3, 2017.

[37] J.A. Miller, P.J. Cargill, G. Emslie, G.D. Holman, B.R. Dennis, T.N. LaRosa, R.M. Winglee, S.G. Benka, S. Tsuneta, "Critical issues for understanding particle acceleration in impulsive solar flares”, Journal of Geophysical Research, Vol. 102, No. A7, Pages 1463114659, 1997.

[38] E.R. Priest, T.G. Forbes, "The magnetic nature of solar flares”, The Astronomy and Astrophysics Review, 10, 313-377, 2002.

[39] E.N. Parker, "The solar flare phenomenon and theory of reconnection and annihilation of magnetic field", ApJS, 8, 1963.

[40] Charles L. Wolff, "Free oscillations of the Sun and their possible stimulation by solar flares", The Astrophysical Journal, 176, 833$842,1972$.

[41] C. Karoff, H Jeldsen, "Evidence that solar flares drive global oscillations in the Sun", The Astrophysical Journal, 678, L73-L76, 2008.

[42] Brajesh Kumar, Savita Mathur, Rafael A. Garcia, Antonio Jimenez, "On the signatures of flare-induced global waves in the Sun: GOLF and VIRGO observations", arXiv: 1710.06245v1 [astro-ph.SR], 2017.

[43] T.G. Cowling, "Chapter 8: Solar electrodynamics", Sun, edited by G.P. Kuiper, University of Chicago Press, 1953

[44] J. W. Dungey, "The motion of magnetic fields", MNRAS, 113, 1953.

[45] P.A. Sweet, "The neutral point theory of flares", IAUS, 6, 1958.

[46] J. Heyvaerts, E.R. Priest, D.M. Rust, “An emerging flux model for solar flare phenomenon”, The Astrophysical Journal, 216, 123137, 1977.

[47] S. Tsuneta, "Structure and dynamics of magnetic reconnection in a solar flare", The Astrophysical Journal, 456, 840-849, 1996.

[48] Huge Carmichael, "A process for flares", NASSP, 50, 451C, 1964.

[49] T. Hirayama, "Theoretical model for flares and prominences I. Evaporating Flare Model", Solar Physics, 34, 323-338, 1974.

[50] P. A. Sturrock, "Model of the high-energy phase of solar flare", Nature, 211, 695-697, 1996. 
An Insight into Space Weather

[51] R.A. Kopp, G.W. Pneuman, "Magnetic reconnection in the corona and the loop prominence phenomenon", Solar Physics, 50, 85-98, 1976.

[52] Arnold O. Benz, "Flare Observations", Living Reviews in Solar Physics, 14:2, 2017.

[53] S.F. Martin, "Preflare conditions, changes and events", Solar Physics, 68, 217-236, 1980.

[54] L. Fletcher, B.R. Dennis, H.S. Hudson, S. Krucker, K. Phillips, A. Veronig, M. Battaglia, L. Bone, A. Caspi, Q. Chen, P. Gallagher, P.T. Grigis, H. Ji, W. Liu, R.O. Milligan, M. Temmer, "An observational overview of the solar flare”, arxiv: 1109.5932v2, 2011.

[55] T.G. Forbes, L.W. Acton, "Reconnection and field line shrinkage in a solar flare", The Astrophysical Journal, 459, 330-341, 1996.

[56] Seiji Yashiro, Nat Gopalswamy, "Statistical relationship between solar flares and coronal mass ejections", Universal Heliophysical Processes, Proceedings IAU Symposium no. 257”, 2008.

[57] J. Zhang, K.P. Dere, R.A. Howard, A. Vourlidas, "A study of the kinematic evolution of coronal mass ejection", The Astrophysical Journal, 604, 420-432, 2004.

[58] M. Dryer, S. Cuperman, "The solar wind: A review", saop.book, 197D, 1976.

[59] Steven R Cranmer, "Origins of the Ambient Solar Wind: Implications for space weather", arXiv:1708.07169v1 [astro-ph.SR], 2017.

[60] L. Biermann, "Physical processes in comet tails and their relations to solar activity", LIACo, 4, 1952.

[61] E.N. Parker, "Dynamics of the interplanetary gas and magnetic fields", The Astrophysical Journal, 128, 1958.

[62] E.N. Parker, "The solar wind", Journal of research of the National Bureau of Standards - D Radio propagation, Vol. 65D, No. 6, 537-542, 1961.

[63] P.J. Coleman Jr., "Turbulence, viscosity and dissipation in the solar wind plasma", The Astrophysical Journal, Vol. 153, 371-388, 1968.

[64] E.N. Parker, "Theoretical studies of the solar wind phenomenon", Space Science Reviews, 9, 325-360, 1969.

[65] M.L. Goldstein, D.A. Roberts, "magnetohydrodynamic turbulence in the solar wind", Annual Review in Astronomy and Astrophysics, 33, 283-325, 1995

[66] A.S. Krieger, A.F. Timothy, E.C. Roelof, “A coronal hole and its identification as the source of a high velocity solar wind stream”, Solar Physics, 29, 505-525, 1973.

[67] J.T. Nolte, A.S. Krieger, A.F. Timothy, R.E. Gold, E.C. Roelof, G. Vaiana, A.J. Lazarus, J.D. Sullivan, P.S. McIntosh, “Coronal holes as sources of solar wind", Solar Physics, 46, 303-322, 1976.

[68] Chuan-yi Tu, Cheng Zhou, Eckart Marsch, Li-Dong Xia, Liang Zhao, Jing-Xiu Wang, Klauss Wilhelm, "Solar wind origin in coronal funnels", Science, Vol. 308, Issue 5721, 519-523, 2005.

[69] J.F. McKenzie, M. Banaszkiewicz, W.I. Axford, “Acceleration of the high speed solar wind”, Astronomy and Astrophysics, 303, 4548, 1995.

[70] S.R. Cranmer, A.A. VanBallegooijen, R.J. Edgar, "Self-consistent coronal heating and solar wind acceleration from anisotropic magnetohydrodynamic turbulence", The Astrophysical Journal Supplement Series, 171, 520-551, 2007.

[71] B. De Pontieu, S.W. McIntosh, M. Carlsson, V.H. Hansteen, T.D. Tarbell, C.J. Schrijver, A.M. Title, R.A. Shine, S. Tsuneta, Y. Katsukawa, K. Ichimoto, Y. Seumatsu, T. Shimizu, S. Nagata, "Chromospheric Alfvenic waves strong enough to power the solar wind", Science, 318, 1574- 1577, 2007.

[72] S.W. McIntosh, B. De Pontieu, M. Carlsson, V. Hansteen, P. Boerner, M. Goossens, "Alfvenic waves with sufficient energy to power the quiet solar corona and fast solar wind", Nature, 475, 477-480, 2011.

[73] Y. -M, Wang, "Small coronal holes near active regions as sources of slow solar winds", The Astrophysical Journal, 841:94 (18pp), 2017.

[74] J. R. Jokipii, "Propagation of cosmic rays in the solar wind", Reviews of Geophysics and Space Physics, Vol. 9, No. 1, $27-87,1971$.

[75] V.B. Baranov, Y.G. Malama, "Model of the solar wind interaction with the local interstellar medium: Numerical solution of selfconsistent problem", Journal of Geophysical Research, Vol. 98, No. A9, 157-164, 1993.

[76] G.P. Zank, "Interaction of the solar wind with the local interstellar medium; a theoretical perspective", Space Science Reviews, 89, 413-688, 1999.

[77] L. Rezeau, "chapter 1: Discontinuities and turbulence in the solar wind", The environment of the sun and the stars, Lecture notes in physics 857, Springer, 2013.

[78] S.-I. Akasofu, "Energy coupling between the solar wind and the magnetosphere", Space Science Reviews, 28, 121, 1981.

Publish your research article in AIJR journals-

Online Submission and Tracking

$\checkmark$ Peer-Reviewed

$\checkmark$ Rapid decision

$\checkmark$ Immediate Publication after acceptance

$\checkmark \quad$ Articles freely available online

$\checkmark \quad$ Retain full copyright of your article.

Submit your article at journals.aijr.in
Publish your books with AIJR publisher-

$\checkmark$ Publish with ISBN and DOI.

$\checkmark$ Publish Thesis/Dissertation as Monograph.

$\checkmark$ Publish Book Monograph.

$\checkmark$ Publish Edited Volume/ Book.

$\checkmark$ Publish Conference Proceedings

$\checkmark \quad$ Retain full copyright of your books.

Submit your manuscript at books.aijr.org 